\title{
Structural Robustness and Multi-Model Control in Gap Metric
}

\author{
Duan Yubo, Yao Sai and Liu Bin \\ School of Electrical and Information Engineering \\ liubinnepu@gmail.com
}

\begin{abstract}
The gap metric is a successful metric in control theory, which can measure the uncertainty and describe the performance specifcations of the robust control system. In the framework of this metric, robust stability radius is proposed to characterize the stability robustness of the closed-loop system. When both the plant and the controller have uncertainties simultaneously, we introduce the structural robust stability, and prove the necessary and sufficient conditions of the robust stability of the feedback control system. In this paper, we proposed a multi-model robust control method, where the robust stability radius is used to generate the weights. Last, the simulation of a typical nonlinear process can prove the valid of the new strategy.
\end{abstract}

Keywords: Structural robustness; nullity; deficiency; multi-model control; gap metric

\section{Introduction}

Feedback control can handle uncertainty. The extent of closed-loop system to tolerate uncertainty before the whole system becomes unstable is called closed-loop robustness [1]. If the closed-loop system $[P, C]$ is stable, when the plant $P$ is perturbed to be $P_{1}$, and the perturbed closed-loop system $\left[P_{1}, C\right]$ is also stable, we say the feedback system $[P, C]$ has robust stability. By introducing some appropriate metric $d(\cdot, \cdot)$, when $d\left(P_{1}, P\right)<\varepsilon$, the closed-loop system $\left[P_{1}, C\right]$ is stable. In this case, the main advantage is that we need not any priori knowledge of $\left[P_{1}, C\right]$, but a suitable metric.

The traditional robust control theory is based on $\infty$-norm, which has become a fairly perfect systematic theory. However, $\infty$-norm can only be used to measure the distance between two stable systems not the unstable systems. Sometimes, it is not appropriate to measure differences of two stable systems. The gap metric was first introduced into the control theory by El-Sakkary [2]. After that, $v$-gap metric and graph metric are proposed by Vinnicombe [3] and Vidyasagar [4], respectively. Another important one is the pointwise gap metric [5]. In this paper, we discuss the classical gap metric mainly.

The linear system theory has been well developed, where the performances and the robustness of the linear system can be met completely. However, the linear controller may lead to serious performance limitations when applied to nonlinear systems, especially in whole operating range. It is well-known that the nonlinear controller is not necessarily for a nonlinear plant. One hand, the feedback control itself can generate a certain degree of linearizing effect. On the other hand, some advanced remedies can overcome this problem, such as gain scheduling, adaptive control, and so on [6]. Multi-model control of nonlinear system is another important approach for nonlinear plant [7]. And it has become an active 
research field of the nonlinear system control theory in the last few years. In practical engineering applications, the multi-model method has applied with success, particularly in adaptive control scheme [8,9], where the local linear models are obtained in some operating points. In this method, the nonlinear system is converted into the combination of the linear models, and local linear controllers according to local linear models are designed firstly. Then the actions of the local controllers constitute the total output of the global controller, which will be implemented on the nonlinear plant. At most time, the actual output of the nonlinear plant is different from the outputs of the local linear models of various operating point. The differences are often used to generate the weights [10].

In this paper, we will answer such an interesting question: "When is a linear controller sufficient to control a nonlinear process?" And the organization of this paper is as follows: In Section 2, the gap metric is introduced briefly; In Section 3, the robust stability radius is defined and the significance of the optimal robust controller is analyzed; In Section 4, the robust stability of the closed-loop system is considered, and the corresponding necessary and sufficient condition is proved where two important concepts of the operator, "nullity" and "deficiency", are introduced; a new multi-model approach is proposed in Section 5, where a global controller is designed for a nonlinear process and $b_{P, C}$ used to produce the weights of the local multilinear controllers; At last, an example is adopted to illustrated the effective of the new method.

\section{Gap Metric}

Most control system designs are based on mathematical models. However, there is no single fixed model can represent the true plant exactly. The quality of a model depends on how closely its responses match those of the physical system. A real system can be described as an uncertainty model set with nominal model. In this paper, the model set is defined in the gap metric.

Consider a standard feedback system configuration consisting of a system $P \in \mathrm{P}^{l \times m}$ and a controller $C \in \mathrm{P}^{m \times l}$ denoted by $[P, C]$, as depicted in Figure 1, where $\mathrm{P}^{i \times j}$ denotes the set of all $i \times j$ matrices with elements of real rational transfer functions.

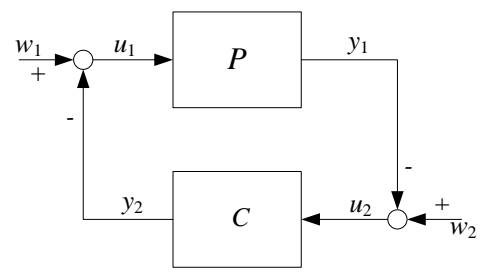

Figure 1. Example of a figure caption

Taking the plant $P$ as an operator

$$
P: \mathrm{D}_{P} \subset \mathrm{X} \rightarrow \mathrm{Y},
$$

where

$$
\mathrm{D}_{P}:=\left\{u \in \mathrm{X} \subset \mathrm{H}_{2}: P u \in \mathrm{Y} \subset \mathrm{H}_{2}\right\}
$$

is the domain of $P, \mathrm{X}, \mathrm{Y}$ are the input subspace and the output subspace, respectively. Similarly, the controller $C$ can also be regarded as the operator, 


$$
C: \mathrm{D}_{C} \subset \mathrm{Y} \rightarrow \mathrm{X} .
$$

The graph of the plant is defined as

$$
\mathrm{G}_{P} \square\left\{\left[\begin{array}{c}
P u \\
u
\end{array}\right]: u \in \mathrm{D}_{P}\right\}=\left[\begin{array}{c}
P \\
I_{m}
\end{array}\right] \mathrm{D}_{P} \subset \mathrm{Y} \oplus \mathrm{X} .
$$

Similarly, the graph of the controller can be defined as

$$
\mathrm{G}_{C} \square\left\{\left[\begin{array}{c}
u \\
C u
\end{array}\right]: u \in \mathrm{D}_{C}\right\}=\left[\begin{array}{l}
I_{l} \\
C
\end{array}\right] \mathrm{D}_{C} \subset \mathrm{X} \oplus \mathrm{Y} .
$$

The inverse graph of the controller is defined as

$$
\mathrm{G}_{C}^{I} \square\left\{\left[\begin{array}{c}
C u \\
u
\end{array}\right]: u \in \mathrm{D}_{C}\right\}=\left[\begin{array}{c}
C \\
I_{l}
\end{array}\right] \mathrm{D}_{C}=\left[\begin{array}{ll}
0 & I \\
I & 0
\end{array}\right] \mathrm{G}_{C} 。
$$

If the nominal plant $P_{1}$ is perturbed to $P_{2}$, then the distance between $P_{1}$ and $P_{2}$ is defined

$$
\delta\left(P_{1}, P_{2}\right)=\delta\left(\mathrm{G}_{P_{1}}, \mathrm{G}_{P_{2}}\right) .
$$

The uncertainty model set is defined in gap metric

$$
\mathrm{B}\left(P_{1}, r\right) \square\left\{P_{2} \mid \delta\left(P_{1}, P_{2}\right)<r\right\},
$$

where $P_{1}$ is the center of the "gap ball", and $r$ is the "radius".

The gap can be computed by the direct gap [1]:

$$
\delta\left(P_{1}, P_{2}\right)=\max \left\{\vec{\delta}\left(\mathrm{G}_{P_{1}}, \mathrm{G}_{P_{2}}\right), \vec{\delta}\left(\mathrm{G}_{P_{2}}, \mathrm{G}_{P_{1}}\right)\right\},
$$

where

$$
\begin{aligned}
& \vec{\delta}\left(\mathrm{G}_{P_{1}}, \mathrm{G}_{P_{2}}\right)=\sup _{u_{1} \in \mathrm{D}_{\mathrm{A}}, u_{1} \neq 0} \inf _{u_{2} \in \mathrm{D}_{P_{2}}} \frac{\left\|u_{1}-u_{2}\right\|^{2}+\left\|P_{1} u_{1}-P_{2} u_{2}\right\|^{2}}{\sqrt{\left\|u_{1}\right\|^{2}+\left\|P_{1} u_{1}\right\|^{2}}}, \\
& \vec{\delta}\left(\mathrm{G}_{P_{2}}, \mathrm{G}_{P_{1}}\right)=\sup _{u_{2} \in \mathrm{D}_{P_{2}}, u_{2} \neq 0} \inf _{u_{1} \in \mathrm{D}_{\mathrm{P}_{1}}} \frac{\left\|u_{2}-u_{1}\right\|^{2}+\left\|P_{2} u_{2}-P_{1} u_{1}\right\|^{2}}{\sqrt{\left\|u_{2}\right\|^{2}+\left\|P_{2} u_{2}\right\|^{2}}} .
\end{aligned}
$$

If $\delta\left(P_{1}, P_{2}\right)<1$, then

$$
\delta\left(P_{1}, P_{2}\right)=\vec{\delta}\left(\mathrm{G}_{P_{1}}, \mathrm{G}_{P_{2}}\right)=\vec{\delta}\left(\mathrm{G}_{P_{2}}, \mathrm{G}_{P_{1}}\right) .
$$

It is well known that each real rational matrix has both right-coprime and left-coprime factorizations over the ring of all stable real rational functions [4]. Let $\left(D_{1}, N_{1}\right)$, $\left(D_{2}, N_{2}\right)$ denote the normalized right coprime factorizations of $P_{1}$ and $P_{2}$, respectively, then

$$
\delta\left(P_{1}, P_{2}\right)=\delta\left(\mathrm{G}_{P_{1}}, \mathrm{G}_{P_{2}}\right)=\inf _{Q \in H_{\infty}}\left\|\left[\begin{array}{l}
N_{1} \\
D_{1}
\end{array}\right]-\left[\begin{array}{c}
N_{2} \\
D_{2}
\end{array}\right] Q\right\| .
$$


Let $\left(D_{P}, N_{P}\right),\left(\tilde{N}_{P}, \tilde{D}_{P}\right)$ denote a normalized right coprime factorization and a normalized left coprime factorization of the plant, respectively. Similarly, $\left(D_{C}, N_{C}\right)$ and $\left(\tilde{N}_{C}, \tilde{D}_{C}\right)$ will denote a normalized right and a left factorizations of the compensator $C$. We can define

$$
\begin{gathered}
G_{P} \square\left[\begin{array}{l}
D_{P} \\
N_{P}
\end{array}\right], \tilde{G}_{P} \square\left[\begin{array}{ll}
-\tilde{N}_{P} & \tilde{D}_{P}
\end{array}\right], \\
G_{C} \square\left[\begin{array}{l}
N_{C} \\
D_{C}
\end{array}\right], \tilde{G}_{C} \square\left[\begin{array}{ll}
\tilde{D}_{C} & -\tilde{N}_{C}
\end{array}\right] .
\end{gathered}
$$

where $G_{P}, \tilde{G}_{P}$ are called normalized right, left graph symbols of $P$, and $G_{C}, \tilde{G}_{C}$ normalized right, left graph symbols of $C$. Note that

$$
\tilde{G}_{P} G_{P}=\tilde{G}_{C} G_{C}=0,
$$

and so $\left[\begin{array}{ll}G_{P} & \tilde{G}_{P}^{*}\end{array}\right]$ and $\left[\begin{array}{ll}G_{C} & \tilde{G}_{C}^{*}\end{array}\right]$ are unitary.

\section{Optimal Robust Control}

By far, one of the most common ways to assess closed-loop robustness is to examine the sensitivity function of the system with respect to noise and disturbances [11]. As depicted in Figure 1, this dependence can be written as follows:

$$
\left[\begin{array}{l}
u_{1} \\
y_{1}
\end{array}\right]=\left[\begin{array}{l}
I \\
P
\end{array}\right](I-C P)^{-1}\left[\begin{array}{ll}
I & -C
\end{array}\right]\left[\begin{array}{l}
w_{1} \\
w_{2}
\end{array}\right],
$$

or

$$
\left[\begin{array}{l}
u_{1} \\
y_{1}
\end{array}\right]=\left[\begin{array}{cc}
(I-C P)^{-1} & -(I-C P)^{-1} C \\
P(I-C P)^{-1} & -P(I-C P)^{-1} C
\end{array}\right]\left[\begin{array}{l}
w_{1} \\
w_{2}
\end{array}\right] .
$$

If the closed-loop is stable, we are interested in minimizing the effects of $\left[\begin{array}{l}w_{1} \\ w_{2}\end{array}\right]$ to $\left[\begin{array}{l}u_{1} \\ y_{1}\end{array}\right]$. This means that we need to minimize the following cost function

$$
\rho \square\left\|\left[\begin{array}{l}
I \\
P
\end{array}\right](I-C P)^{-1}\left[\begin{array}{ll}
I & -C
\end{array}\right]\right\|_{\infty},
$$

which can be alternatively defined as:

$$
b_{P, C} \square \frac{1}{\rho}=\left\|\left[\begin{array}{l}
I \\
P
\end{array}\right](I-C P)^{-1}\left[\begin{array}{ll}
I & -C
\end{array}\right]\right\|_{\infty}^{-1} .
$$

$b_{P, C}$ is called the robust stability radius, which can be regarded as the generalized stability margin. It is firstly introduced by T.T. Georgiou and M.C. Smith directly in terms of the plant and the controller [12].

If both $P$ and $C$ are stable, the $(I, P)$ and $(C, I)$ can be regarded as the right coprime factorization and left coprime factorization of $P$ and $C$, respectively. 
The equation (7) can also be stated as

$$
\left[\begin{array}{l}
u_{1} \\
y_{1}
\end{array}\right]=\left[\begin{array}{ll}
D_{P} \Delta^{-1} \tilde{D}_{C} & -D_{P} \Delta^{-1} \tilde{N}_{C} \\
N_{P} \Delta^{-1} \tilde{D}_{C} & -N_{P} \Delta^{-1} \tilde{N}_{C}
\end{array}\right]\left[\begin{array}{l}
w_{1} \\
w_{2}
\end{array}\right],
$$

where $|\Delta(P, C)|$ is the characteristic determinants of the transfer matrix,

$$
\Delta(P, C)=\tilde{D}_{C} D_{P}-\tilde{N}_{C} N_{P}=\tilde{G}_{C} G_{P} .
$$

If the closed-loop system is stable, according the definition of $G_{P}, \tilde{G}_{C}$, equation (9) can be written in a concise manner

$$
b_{P, C}=\left\|G_{P} \tilde{G}_{C}\right\|_{\infty}^{-1} .
$$

Note that the value of $b_{P, C}$ is always between 0 and 1 , and small $b_{P, C}$ means that the closedloop stability margin is small. When $b_{P, C}=0$, the closed-loop system will become unstable. Larger $b_{P, C}$ indicates that the system has good robustness.

The optimal robust stabilization problem is to maximize the robust stability radius $b_{P, C}$, i.e.,

$$
b^{*}(P) \square\left\{\inf _{C}\left\|G_{\text {stabilizing }} \tilde{G}_{P}\right\|_{\infty}\right\}^{-1} .
$$

The corresponding controller is called the optimal robust controller.

\section{Structural Robustness of Closed-Loop System}

Consider the Figure 1, there will be

$$
\left[\begin{array}{l}
w_{1} \\
w_{2}
\end{array}\right]=\underbrace{\left[\begin{array}{ll}
P & I \\
I & C
\end{array}\right]}_{J(P, C)}\left[\begin{array}{l}
u_{1} \\
u_{2}
\end{array}\right] .
$$

If the transfer matrix from $\left[\begin{array}{l}w_{1} \\ w_{2}\end{array}\right]$ to $\left[\begin{array}{l}u_{1} \\ y_{1}\end{array}\right]$ has the unique solution and physical realization, then $J^{-1}(P, C)$ exists. The closed-loop system is stable when $J^{-1}(P, C)$ is bounded. This means:

$$
\left[\begin{array}{c}
w_{1} \\
w_{2}
\end{array}\right]=\underbrace{\left[\begin{array}{c}
P \\
I
\end{array}\right]}_{\mathrm{W}} u_{1}+\underbrace{\left[\begin{array}{c}
I \\
C
\end{array}\right] u_{2},}_{\mathrm{G}_{\mathrm{C}}^{\prime}}
$$

That is $\mathrm{W}=\mathrm{G}_{p} \oplus \mathrm{G}_{C}^{I}$.

Lemma 1[13] The feedback system $[P, C]$ is stable if and only if

(i) $\mathrm{G}_{P} \cap \mathrm{G}_{C}^{I}=\{0\}$,

(ii) $\mathrm{W}=\mathrm{G}_{P} \oplus \mathrm{G}_{C}^{I}$. 
In other words, the graph of the plant and the inverse graph of the controller have induced a coordinatization of $\mathrm{W}$ :

The unique decomposition of $\mathrm{W}=$ means that there exists a pair of projection operators. From Figure 1, the transfer functions from $\left[\begin{array}{l}w_{1} \\ w_{2}\end{array}\right]$ to $\left[\begin{array}{l}y_{1} \\ u_{1}\end{array}\right],\left[\begin{array}{l}u_{2} \\ y_{2}\end{array}\right]$ are

$$
\begin{aligned}
& H_{P}=\left[\begin{array}{l}
P \\
I
\end{array}\right](I-C P)^{-1}\left[\begin{array}{ll}
-C & I
\end{array}\right], \\
& H_{C}=\left[\begin{array}{l}
I \\
C
\end{array}\right](I-P C)^{-1}\left[\begin{array}{ll}
I & -P
\end{array}\right],
\end{aligned}
$$

respectively.

Because of $H_{P}^{2}=H_{P}$ and $H_{C}^{2}=H_{C}, H_{P}$ and $H_{C}$ can be regarded as the projection operators from $\mathrm{W}$ : to $\mathrm{G}_{P}$ and $\mathrm{G}_{C}^{I}$, respectively. Particularly, any signal in $\mathrm{W}$ space can be uniquely projected to $\mathrm{G}_{P}$ and $\mathrm{G}_{C}^{I}$ since $H_{P}+H_{C}=I$. The corresponding geometric interpretation is that $H_{P}$ is the parallel projection operator along $\mathrm{G}_{C}^{I}$ to $\mathrm{G}_{P}$, and $H_{C}$ the parallel projection operator along $\mathrm{G}_{P}$ to $\mathrm{G}_{C}^{I}$, denoted by $\Pi_{\mathrm{G}_{p} \subset \mathrm{G}_{\mathrm{C}}^{l}}$ and $\Pi_{\mathrm{G}_{\mathrm{C}}^{l}\left[\mathrm{G}_{p}\right.}$, respectively.

It should be noted that parallel projection operators $\Pi_{G_{p}\left\lceil G_{t}^{l}\right.}$ and $\Pi_{G_{C}^{l}\left\lceil G_{p}\right.}$ can not be applied to nonlinear system. This is because

$$
\begin{aligned}
& \Pi_{\mathrm{G}_{p} \square \complement_{C}^{l}}=\left[\begin{array}{c}
P \\
I
\end{array}\right](I-C P)^{-1}\left[\begin{array}{ll}
-C & I
\end{array}\right]=\left[\begin{array}{cc}
0 & 0 \\
0 & I
\end{array}\right]+\left[\begin{array}{cc}
I & 0 \\
0 & -I
\end{array}\right]\left[\begin{array}{cc}
P & I \\
I & C
\end{array}\right]^{-1}, \\
& \Pi_{\mathrm{G}_{E}^{l} \backslash G_{p}}=\left[\begin{array}{c}
I \\
C
\end{array}\right](I-P C)^{-1}\left[\begin{array}{ll}
I & -P
\end{array}\right]=\left[\begin{array}{ll}
I & 0 \\
0 & 0
\end{array}\right]+\left[\begin{array}{cc}
-I & 0 \\
0 & I
\end{array}\right]\left[\begin{array}{ll}
P & I \\
I & C
\end{array}\right]^{-1} .
\end{aligned}
$$

Hence, we introduce "nullity" and "deficiency" to analyze the stability and robust stability of the closed-loop system.

Let $\mathrm{X}, \mathrm{Y}$ be subspaces of $\mathrm{G}^{n}$, nullity and deficiency are defined as:

$$
\operatorname{nul}(X, Y):=\operatorname{dim}(X \cap Y), \operatorname{def}(X, Y):=\operatorname{cod} \operatorname{din}(X+Y),
$$

where $\operatorname{dim}(\mathrm{G})$ denotes the dimension of the space $\mathrm{G}$.

Therorem 1 The feedback system $[P, C]$ is stable if and only if

(i) $\operatorname{nul}\left(\mathrm{G}_{P}, \mathrm{G}_{C}^{I}\right)=0$,

(ii) $\operatorname{def}\left(\mathrm{G}_{P}, \mathrm{G}_{C}^{I}\right)=0$.

Proof: $(\Leftarrow)$ 
By the definitions of $\operatorname{nul}(\cdot, \cdot)$ and $\operatorname{def}(\cdot, \cdot)$, we can get immediately $\operatorname{def}\left(\mathrm{G}_{P}, \mathrm{G}_{C}^{I}\right)=0$ means $\mathrm{W}=\mathrm{G}_{p}+\mathrm{G}_{C}^{I}$, and $\operatorname{nul}\left(\mathrm{G}_{p}, \mathrm{G}_{C}^{I}\right)=0$ means $\mathrm{G}_{p} \cap \mathrm{G}_{C}^{I}=\{0\}$. From Lemma 1, the feedback system $[P, C]$ is stable.

$(\Rightarrow)$

If $\operatorname{nul}\left(\mathrm{G}_{P}, \mathrm{G}_{C}^{I}\right) \neq 0$, that is $\mathrm{G}_{P} \cap \mathrm{G}_{C}^{I} \neq\{0\}, \Pi_{\mathrm{G}_{p} \sqcap C_{C}^{l}}$ and $\Pi_{\mathrm{G}_{\mathrm{C}}^{l} \square \mathrm{G}_{p}}$ are not uniqueness. By Lemma 1 , the feedback system $[P, C]$ is unstable, which contradicts to the condition. So, $\operatorname{nul}\left(\mathrm{G}_{p}, \mathrm{G}_{C}^{I}\right)=0$. Similarly, if $\operatorname{def}\left(\mathrm{G}_{p}, \mathrm{G}_{C}^{I}\right) \neq 0$, there will be $\mathrm{G}_{p} \oplus \mathrm{G}_{C}^{I} \subset \mathrm{W}$ =, which contradicts to $\mathrm{W}=\mathrm{G}_{p} \oplus \mathrm{G}_{C}^{I}$. Therefore, $\operatorname{def}\left(\mathrm{G}_{p}, \mathrm{G}_{C}^{I}\right)=0$.

This is the end of the proof.

In robust control theory, the controller should have enough tunable margin in order to satisfy different performance specifications, i.e., the designed controller should have some robustness. So, uncertainties of both the plant and the controller should be considered in order that the controller has some robustness. It is meaningful to study the robust control problem when both the plant and the controller have uncertainties. Therefore, the robustness is called "structural robustness", because of the plant and the controller having uncertainties simultaneously.

In this section, we first give an important lemma before the closed-loop robust stability theorem.

Lemma 2[14] Let $\mathrm{X} \in \mathrm{G}_{m}^{n}, \mathrm{Y} \in \mathrm{G}_{n-m}^{n}, \rho$ be the unitarily invariant angular metric defined by symmetric gauge function. When $\rho(\mathrm{X}, \tilde{\mathrm{X}}) \leq \alpha, \rho(\mathrm{Y}, \tilde{\mathrm{Y}}) \leq \beta, \alpha>0, \beta>0$, $1 \leq k \leq \min \{m, n-m\}$,

(i) $\operatorname{nul}(\tilde{X}, \tilde{Y})<k$ if and only if

$$
\alpha+\beta<\mathrm{F}\left[0, \cdots, 0, \theta_{m-k+1}(\mathrm{X}, \mathrm{Y}), \cdots, \theta_{\min \{m, n-m\}}(\mathrm{X}, \mathrm{Y})\right]
$$

(ii) $\operatorname{def}(\tilde{X}, \tilde{Y})<k$ if and only if

$$
\alpha+\beta<\mathrm{F}\left[0, \cdots, 0, \theta_{m-k+1}\left(\mathrm{X}^{\perp}, \mathrm{Y}^{\perp}\right), \cdots, \theta_{\min \{m, n-m\}}\left(\mathrm{X}^{\perp}, \mathrm{Y}^{\perp}\right)\right]
$$

Theorem 2 The feedback system $[P, C]$ is stable, where $P \in \mathrm{P}^{l \times m}$ and $C \in \mathrm{P}^{m \times l}$ are the nominal plant and the nominal controller, respectively. When both the plant and the controller have uncertainties in gap metric

$$
P_{1}(j \omega) \in \mathrm{B}\left[P(j \omega), r_{P}\right], C_{1}(j \omega) \in \mathrm{B}\left[C(j \omega), r_{C}\right],
$$

$\left[P_{1}, C_{1}\right]$ is stable if and only if

$$
r_{P}+r_{C}<b_{P, C} \cdot
$$

Proof: Without loss of generality, let $m<l$.The graph of the plant and the inverse graph of the controller are denoted by $\mathrm{G}_{p}$ and $\mathrm{G}_{c}^{l}$, respectively, then $\mathrm{G}_{p} \in \mathrm{G}_{m}^{n}, \mathrm{G}_{C}^{l} \subset \mathrm{G}^{n}$. 
Let $\delta$ be the gap metric. When both the plant and the controller have uncertainties, the graph and the inverse graph denoted by $\mathrm{G}_{p_{1}} \in \mathrm{G}_{m}^{n}$ and $\mathrm{G}_{C_{1}}^{l} \subset \mathrm{G}_{1}^{n}$ satisfying

$$
\delta\left(\mathrm{G}_{P}, \mathrm{G}_{P_{1}}\right) \leq r_{P}, \delta\left(\mathrm{G}_{C}^{I}, \mathrm{G}_{C_{1}}^{I}\right) \leq r_{C} .
$$

By Theorem 1, the perturbed system is stable if and only if

$$
\operatorname{nul}\left(\mathrm{G}_{P_{1}}, \mathrm{G}_{C_{1}}^{I}\right)=0, \operatorname{def}\left(\mathrm{G}_{P_{1}}, \mathrm{G}_{C_{1}}^{I}\right)=0 .
$$

Let $k=1$. From the definition of the gap metric and the angular metric, by Lemma 1 we know

$$
\operatorname{nul}\left(\mathrm{G}_{P_{1}}, \mathrm{G}_{C_{1}}^{I}\right)=0 \text { and } \operatorname{def}\left(\mathrm{G}_{P_{1}}, \mathrm{G}_{C_{1}}^{I}\right)=0
$$

if and only if

$$
\begin{gathered}
r_{P}+r_{C}<\sin \mathrm{F}\left[0, \cdots, 0, \theta_{\min \{m, n-m\}}\left(\mathrm{G}_{P}, \mathrm{G}_{C}^{I}\right)\right], \\
r_{P}+r_{C}<\sin \mathrm{F}\left[0, \cdots, 0, \theta_{\min \{m, n-m\}}\left(\mathrm{G}_{P}^{\perp},\left(\mathrm{G}_{C}^{I}\right)^{\perp}\right)\right] .
\end{gathered}
$$

According to the properties of the canonical angles of subspaces,

$$
\Theta\left(\mathrm{G}_{P}^{\perp},\left(\mathrm{G}_{C}^{I}\right)^{\perp}\right)=\Theta\left(\mathrm{G}_{P}, \mathrm{G}_{C}^{I}\right),
$$

where $\Theta(\mathrm{X}, \mathrm{Y}):=\left(\theta_{1}(\mathrm{X}, \mathrm{Y}), \cdots, \theta_{m}(\mathrm{X}, \mathrm{Y})\right)$ denotes the canonical angles non-increasing ordered between $\mathrm{X}$ and $\mathrm{Y}$.

Obviously, the necessary and sufficient condition of $\operatorname{nul}\left(\mathrm{G}_{P_{1}}, \mathrm{G}_{C_{1}}^{l}\right)=0$ and $\operatorname{def}\left(\mathrm{G}_{P_{1}}, \mathrm{G}_{C_{1}}^{l}\right)=0$ are the same. If symmetric gauge function is selected as the spectral norm,

$$
\mathrm{F}\left[0, \cdots, 0, \theta_{\min \{m, n-m\}}\left(\mathrm{G}_{P}, \mathrm{G}_{C}^{I}\right)\right]=\theta_{\min \{m, n-m\}}\left(\mathrm{G}_{P}, \mathrm{G}_{C}^{I}\right)
$$

Because of

$$
b_{P, C}=\sin \theta_{\min \{m, n-m\}}\left(\mathrm{G}_{P}, \mathrm{G}_{C}^{I}\right),
$$

the closed-loop system $\left[P_{1}, C_{1}\right]$ is stable if and only if

$$
r_{P}+r_{C}<b_{P, C} .
$$

This is the end of the proof.

\section{Multi-Model Control for Nonlinear System}

\subsection{Multi-Model Controller Design}

For a nonlinear system, linearized transfer functions of different equilibrium points over the operating range can be obtained by taking Taylor series approximation. Optimal robust controllers will be designed for every nominal linear model, which can make every linear closed-loop system have largest generalized stability margin. After all local optimal robust 
controllers are constructed; a key issue is how to implement the multi-model control scheme with these local controllers.

Here, considering a set of $m$ local controllers, the global output of the controller can be given by

$$
u(t)=\sum_{i=1}^{m} w_{i}(t) u_{i}(t)
$$

where $u_{i}(t)$ is the output of the $i$-th local controller $C_{i}$, and the $w_{i}(t)$ is the weight coefficient, calculated by

$$
w_{i}=\frac{b_{P_{i}, C_{i}}}{\sum_{j=1}^{m} b_{P_{j}, C_{j}}}, i=1,2, \cdots, m .
$$

Therefore, the multi-mode1 control system structure is depicted in Figure 2.

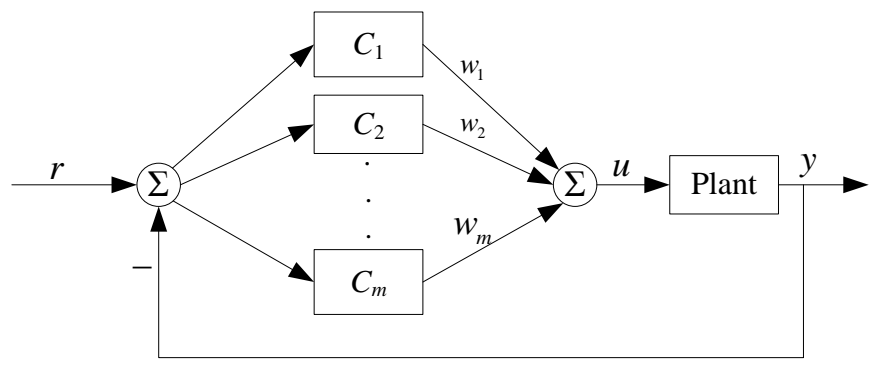

Figure 2. Structure of Multi-Model Control System

\subsection{Process Description and Local Controllers}

Without any loss of generality, an simulation example is adopted from [15]. The plant is a highly nonlinear reactor, whose differential equations are

$$
\begin{aligned}
& \dot{x}_{1}=-x_{1}+D_{a} \cdot r\left(x_{1}, x_{2}\right)-\left(x_{f}-1\right) \\
& \dot{x}_{2}=-x_{2}+B \cdot D_{a} \cdot r\left(x_{1}, x_{2}\right)+\beta\left(u-x_{2}\right) \\
& y=x_{2} \\
& r\left(x_{1}, x_{2}\right)=\left(1-x_{1}\right) \cdot \exp \left(x_{2} /\left(1+x_{2} / \gamma\right)\right)
\end{aligned}
$$

where $x_{1}, x_{2}, u$ and $x_{f}$ are reagent conversion, the dimensionless temperature (output), the dimensionless coolant temperature (input) and dimensionless feed concentration respectively. The nominal values in equations of the constants are $D_{a}=0.072, \gamma=20, B=8, \beta=0.3$, $x_{f}=1$. There are three steady-state points corresponding to input $u=0$.

Three linear transfer functions, optimal robust controllers with their weights at the steady states are given in Table 1. 
Table 1. Local models, local controllers and weights

\begin{tabular}{c|c|c|c}
\hline No. & Linear Models & Models & $w_{i}$ \\
\hline \multirow{2}{*}{ model 1 } & $P_{1}(s)=\frac{0.3 s+0.35}{s^{2}+1.4 s+0.46}$ & $C_{1}(s)=\frac{0.34876 s+0.34676}{s+1.05704}$ & 0.4271 \\
\cline { 3 - 4 } model 2 & $P_{2}(s)=\frac{0.3 s+0.53}{s^{2}+0.36 s-0.41}$ & $C_{2}(s)=\frac{2.589 s+2.213}{s+1.338}$ & 0.1630 \\
\cline { 3 - 4 } model 3 & $P_{3}(s)=\frac{0.3 s+1.26}{s^{2}+1.6 s+1.6}$ & $C_{3}(s)=\frac{0.46672 s+0.46390}{s+1.97773}$ & 0.4099 \\
\hline
\end{tabular}

\subsection{Construction of Global Controller}

The main task of the global controller is to move the reactor operation among the above three steady states. The multi-models are chosen as the three local models given in Table I. These linearized nominal models are controlled using optimal robust controllers, each of which is designed so that every local closed-loop system has largest generalized stability margin. Then the output of the global controller can be calculated according equations (15) and (16).

In Figure 3(a), the set value, outputs of three local linear models and the nonlinear plant are given, denoted by "Set Value", "Output1", "Output2", "Output3" and "Output". Obviously, none of the local linear model can represent the nonlinear system perfectly. The output of the nonlinear plant can track the set value with little fluctuation. It is should be noted that the controller designed around the unstable point (local model $P_{2}$ ) has the largest influence on the closed-loop behavior. The reason is that the second linearized system is unstable, which is the main factor influencing the closed-loop system stability margin.

To study the robustness of the closed-loop system, we put an additive uncertainty on the nonlinear system. The transfer function of the uncertainty is

$$
\Delta(s)=\frac{0.002 s+1}{s+1}
$$

In Figure 3(b), the "Output_Un" denotes the output of the nonlinear plant with uncertainty. To study the behavior of the nonlinear closed-loop system under disturbance rejection, the disturbance is $d=0.2 \sin 2 \pi t$, depicted as Figure 4(a). The closed-loop response is shown in Figure 4(b).

Obviously, the closed-loop system has favorable robustness and disturbance suppressing ability. 


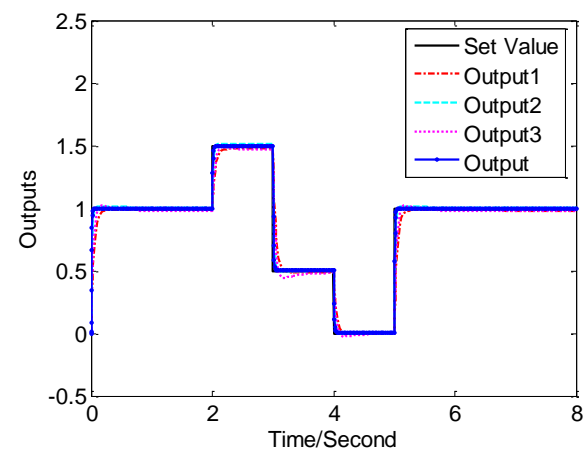

(a) Outputs of local feedback systems and the nonlinear system

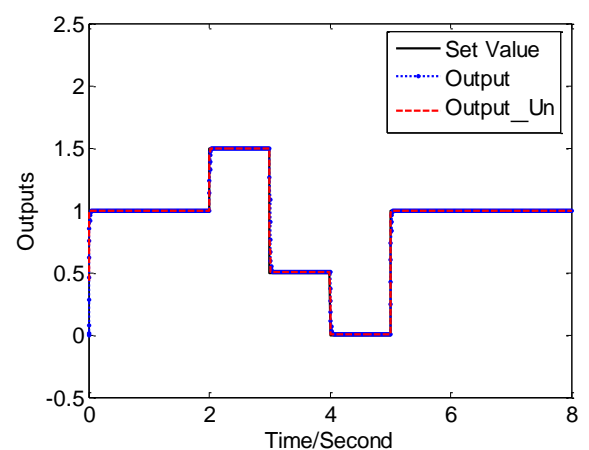

(b) Outputs of the nonlinear system and the perturbed one

Figure 3. Outputs of local feedback systems and the nonlinear system

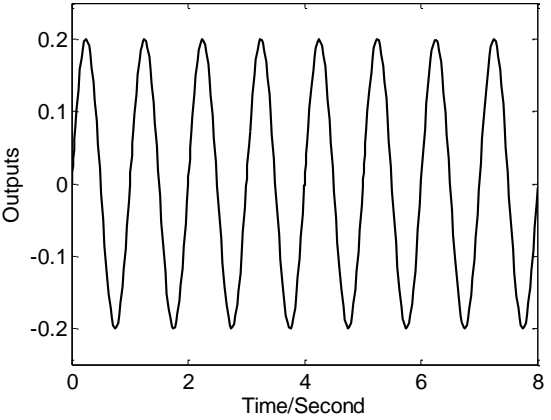

(a) Waveform of disturbance

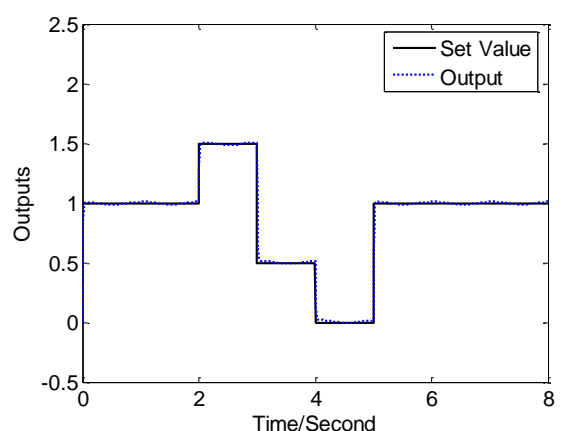

(b) Output of nonlinear system with sine disturbance

Figure 4. Servo response of the nonlinear system

\section{Conclusions}

This paper gives the detailed defination of the gap metric, which can be used to analyze the robust stability, structural robustness, when both the plant and the controller have uncertainties. The robust stability radius is proposed in the gap metric. The nullity and the deficiency are introduced into the robust stability theorem with the robust stability radius. At last, a new multi-model control method for nonlinear system is presented, where the total output of the controller can be obtained by that of the local controller which is weighted in gap metric. The simulations of a highly nonlinear system are studied to illustrate the effective of the new approach, which is superior to [15].

\section{Acknowledgements}

This paper is supported by Youth Science Foundation of Heilongjiang Province (QC2011C043), Youth Academic Backbone Project of Heilongjiang Province, Science and Technology Research Project of Heilongjiang Province (12531058).

\section{References}

[1] K. M. Zhou and J. C. Doyle, "Essentials of Robust Control", Prentice-Hall, Upper Saddle River, (1998).

[2] A. K. El-Sakkary, IEEE Transactions on Automatic Control, vol. 3, no. 30, (1985).

[3] G. Vinnicombe, IEEE Transactions on Automatic Control, vol. 9, no. 38, (1993). 
[4] M. Vidyasagar, "Control System Synthesis: A Factorization Approach”, MIT Press, Cambridge, (1985).

[5] L. Qiu and E. J. Davison, IEEE Transactions on Automatic Control, vol. 6, no. 37, (1992).

[6] W. J. Rugh, IEEE Control Systems Magazine, vol. 1, no. 11, (1991).

[7] R. Murray-Smith and T. A. Johansen, "Multiple model approaches to modeling and control", Taylor \& Francis, London, (1997).

[8] N. S. Kumpati and C. Xiang, IEEE Transaction on Automation Control, vol. 2, no. 42, (1997).

[9] A. Karimi and I. D. Landau, IEEE Transaction on control systems Technology, vol. 2, no. 8, (2000).

[10] A. Banerjee, Y. Arkun, B. Ogunnaike and R. Pearson, American Institute of Chemical Engineers, vol. 5, no. 43, (1997).

[11] L. C. G. J. M. Habets, "Robust Stabilization in the Gap-topology", Springer-Verlag, Berlin, (1991).

[12] T. T. Georgiou and M. C. Smith, IEEE Transaction on Automatic Control, vol. 6, no. 35, (1990).

[13] J. C. Doyle, T. T. Georgiou and M. C. Smith, Systems \& Control Letters, vol. 2, no. 20, (1993).

[14] Y. X. Zhang and L. Qiu, "Linear Algebra and its Applications", vol. 1, (2007), pp. 421.

[15] E. Arslan, M.C. Camurdan, A. Palamglu and Y. Arkun, (Eds.), "Multi-Model Control of Nonlinear Systems Using Closed-Loop Gap Metric", Proceedings of the 2004 American Control Conference, (2004) June 30July 2; Boston, USA. 